\title{
Construction of vertex-disjoint paths in alternating group networks
}

\author{
Shuming Zhou • Wenjun Xiao • Behrooz Parhami
}

Published online: 21 May 2009

(C) The Author(s) 2009. This article is published with open access at Springerlink.com

\begin{abstract}
The existence of parallel node-disjoint paths between any pair of nodes is a desirable property of interconnection networks, because such paths allow tolerance to node and/or link failures along some of the paths, without causing disconnection. Additionally, node-disjoint paths support high-throughput communication via the concurrent transmission of parts of a message. We characterize maximum-sized families of parallel paths between any two nodes of alternating group networks. More specifically, we establish that in a given alternating group network $A N_{n}$, there exist $n-1$ parallel paths (the maximum possible, given the node degree of $n-1$ ) between any pair of nodes. Furthermore, we demonstrate that these parallel paths are optimal or near-optimal, in the sense of their lengths exceeding the internode distance by no more than four. We also show that the wide diameter of $A N_{n}$ is at most one unit greater than the known lower bound $D+1$, where $D$ is the network diameter.
\end{abstract}

Keywords Alternating group graphs · Fault-tolerant routing · Node-disjoint paths · Parallel paths $\cdot$ Robustness $\cdot$ Wide diameter

S. Zhou

Key Laboratory of Network Security and Cryptology, Fujian Normal University, Fuzhou, Fujian 350108, People's Republic of China

S. Zhou

College of Mathematics and Computer Science, Fujian Normal University, Fuzhou, Fujian 350108, People's Republic of China

W. Xiao

Dept. Computer Science, South China University of Technology, Guangzhou, Guangdong 510641, People's Republic of China

B. Parhami $(\bowtie)$

Dept. Electrical and Computer Engineering, University of California, Santa Barbara,

CA 93106-9560, USA

e-mail: parhami@ece.ucsb.edu 


\section{Introduction}

Designers of massively parallel computers, interconnection structures, and networked distributed systems seek desirable attributes that include low node degree, structural regularity, small diameter, rich connectivity, support for simple and efficient routing algorithms, and strong fault tolerance $[6,23,25]$. The efforts to achieve reliability, robustness, maximum concurrency, and minimal transmission delay, exhibited in many recent studies [3, 5-11, 14, 15, 18, 19, 24, 25], will likely intensify as the extent of parallelism and interconnection complexity, both on- and off-chip, continue to increase. Topologies proposed to interconnect the nodes in both parallel and distributed systems have been modeled as graphs, in which the vertices/nodes correspond to processors or routers and the edges/links represent communication channels.

Interestingly, the existence of node-disjoint paths between arbitrary sourcedestination pairs bears on all the important objectives above, given that the existence of such "parallel" paths can help improve reliability, fault tolerance, message throughput, and communication latency. Greater reliability results from tolerance to node and/or link failures along some paths. Improved throughput and communication latency result from the possibility of concurrent transmission of message segments. It is thus of great practical interest to compare various networks with respect to the multiplicity and ease of construction for such parallel paths.

Despite a large collection of widely studied interconnection networks and their associated graphs, each with its proponents and staunch defenders, new networks continue to emerge. It is well known in the parallel and distributed processing community that there is no such thing as a "best" network [20]; assessment of networks with regard to their suitability requires attention to a multitude of structural and performance parameters. A parallel system composed of custom chip-multiprocessors has different bandwidth and latency requirements than a data center or cloud-computing hub with independently operating commodity nodes. Similarly, on-chip [22], system-area [12], and long-haul [13] networks entail different challenges, optimality criteria, and tradeoffs, as do a variety of virtual structures, such as peer-to-peer overlay networks [21].

As we scale up to multimillion-node systems of each type, reliance on commodity interconnects will become increasingly problematic. System performance in such large-scale deployments will likely be limited by communication latency and bandwidth, thus necessitating highly optimized interconnection structures. At such large scales, both richly connected networks (exemplified by high-dimensional $k$-ary $n$ cubes) and hierarchical networks with ad hoc connectivity (clusters and the like) would be unmanageable. Efficient routing decisions and rapid reconfiguration in the event of link and node failures would only be possible if the network in question had systematic properties that could be exploited within the framework of low-complexity and readily scalable distributed algorithms.

Within families of networks that possess desirable uniformity and regularity properties, and hence theoretically tractable (efficient, flexible, and provably correct) distributed algorithms for adaptive routing and reconfiguration, a class based on finite mathematical groups, dubbed Cayley graphs [1], has shown great promise. A wide array of rigorous theoretical results have been obtained for such networks. Among the Cayley graph interconnection architectures studied over the past three decades, the hypercube has drawn the greatest attention, given its many attractive properties, 
including small degree, low diameter, symmetry, strong fault tolerance, and efficient routing algorithms. The hypercube and its numerous modified forms or variants, such as crossed, folded, twisted, and enhanced cubes, have been studied extensively [25]. These classes of networks are collectively known as hypercubic networks. A method to construct $n$ (the maximum possible) vertex-disjoint paths for the $n$-dimensional hypercube $Q_{n}$ has been proposed in [3]. Duh, Chen, and Hsu [8] investigated combinatorial properties of generalized hypercubes, including best containers, wide diameter, and fault diameter. Xu [26] obtained results on the wide diameters of Cartesian product graphs, a class of interconnection networks that includes the hypercune as a special case.

A widely studied alternative to the hypercube, the star graph [2], enjoys most of the desirable properties of the hypercube at considerably lower cost, accommodating more nodes with less interconnection hardware and smaller communication delay. Like the hypercube, the star graph is hierarchically structured and is a member of the class of Cayley graphs. Day and Tripathi [6] identified the $n-1$ (maximum possible) vertex-disjoint paths of the star graph $S_{n}$ and presented a comparative study of $S_{n}$ and $Q_{n}$. Recently, Lin and Duh [19] described a novel routing algorithm for constructing a container of width $n-1$ between any pair of vertices in a generalized star graph, denoted as the $(n, k)$-star graph.

The alternating group network $A N_{n}$, which has a construction similar to the star graph, was proposed by Youhu [27] to improve upon the alternating group graph $A G_{n}$, originally advocated by Jwo, Lakshmivarahan, and Dhall $[16,17]$. Chen, Xiao, and Parhami [4] presented an optimal routing algorithm for the class of alternating group networks. It is the potential advantages of alternating group networks over their better-known brethren that attracts us to them as candidate networks for providing large-scale connectivity in parallel and distributed systems.

In this paper, we expand on the previously known results about $A N_{n}$ by constructing containers of maximum width, deriving the best containers, and computing the wide diameter and fault diameter for $A N_{n}$. The rest of the paper is organized as follows. Section 2 introduces $A N_{n}$, along with some definitions and notation needed for our discussion. Section 3 is devoted to the construction of containers of $A N_{n}$ and the presentation of upper and lower bounds on its wide diameter for $n \geq 4$. Section 4 concludes the paper.

\section{Background and definitions}

This section is devoted to introducing background material and notational conventions needed to understand the rest of the paper. In the study of multiprocessor systems, the topology of a system is often adequately represented by a graph $G=G(V, E)$, where each node $u \in V$ denotes a processor and each edge $(u, v) \in E$ denotes a link between nodes $u$ and $v$. The distance from vertex $u$ to vertex $v$, represented by $d(u, v)$, refers to the length of a shortest path from $u$ to $v$ in $G$. The diameter of $G$, denoted by $D(G)$, is defined as the maximum distance for all pairs of distinct vertices $u$ and $v$ in $G$. For a subset $S \subset V$ of nodes, the notation $G-S$ represents the subgraph obtained by removing the vertices in $S$ from $G$ and also deleting all edges with at least one end vertex in $S$. If $G-S$ is disconnected, then $S$ is called a vertex cut or a separating set. 
The connectivity $\kappa(G)$ of a graph $G(V, E)$, or simply $\kappa$ if the intended graph is unambiguous, is the minimum number of nodes whose removal results in a disconnected or a trivial (one-node) graph. Let $\kappa(G)$ be the connectivity of $G$. According to Menger's theorem, at least $\kappa(G)$ vertex-disjoint paths exist between arbitrary distinct vertices $u$ and $v$ in $G$. The set $C_{K}(u, v)$ of such paths is also called a container of width $\kappa$ between $u$ and $v$. The length $l\left(C_{\kappa}(u, v)\right)$ of $C_{\kappa}(u, v)$ is defined as the length of the longest path in $C_{\kappa}(u, v)$. A best container between $u$ and $v$, denoted by $C_{\kappa}^{*}(u, v)$, is a container of shortest length. Let $d_{\kappa}(u, v)$ be the $\kappa$-wide distance from $u$ to $v$, that is, $d_{\kappa}(u, v)=l\left(C_{\kappa}^{*}(u, v)\right)$. The $\kappa$-wide diameter (or wide diameter) of $G$, denoted by $d_{\kappa}(G)$, is defined as the maximum of $d_{\kappa}(u, v)$ for all pairs of distinct vertices $u$ and $v$ in $G[7,9,14,18,25]$.

Suppose that $H$ is a group and $S$ is a generating set. The Cayley graph $G=$ $\operatorname{Cay}(H, S)$ is constructed as follows: (1) Each element $h$ of $H$ is assigned a vertex, the vertex set $V(G)$ of $G$ is identified with $H$; (2) for any $h \in H$ and $s \in S$, the vertices corresponding to the elements $h$ and $h s$ are joined by a directed edge $(h, h s)$. Thus the edge set $E(G)$ consists of pairs of the form $(h, h s)$. The Cayley graph $G=\operatorname{Cay}(H, S)$ is undirected if the set $S$ is symmetric, i.e. $S=S^{-1}$; the graph $G=\operatorname{Cay}(H, S)$ has no loop if the identity element $e$ of the group $H$ is not in $S$, i.e. $e \notin S$.

Many important and extensively studied interconnection networks, such as the super-torus, hypercube, star graph, and alternating group graph, are Cayley graphs $[1,17]$. Define $\langle n\rangle$ as the set $\{1,2, \ldots, n\}$ and let $p=p_{1} p_{2} \ldots p_{n}$ be a permutation of the elements of $\langle n\rangle$, that is, $p_{i} \in\langle n\rangle$ and $p_{i} \neq p_{j}$ for $i \neq j$. We may refer to $p_{i}$ as $p(i)$, or the $i$ th element of $p$, or use brackets to delineate elements of a permutation when simply juxtaposing them would lead to ambiguities. A permutation $p=p_{1} p_{2} \ldots p_{n}$ of the elements in $\langle n\rangle$ can be represented by its cycle structure, i.e., cyclically ordered sets of symbols with the property that each symbol's desired position is that occupied by the next symbol in the set. For example, the permutation $p=64725831$ consists of the three cycles $C_{1}=(681), C_{2}=(42)$, and $C_{3}=(73)$. Let $p=C_{1} C_{2} \ldots C_{k} e_{1} e_{2} \ldots e_{l}$, where $C_{i}$ is a cycle of length $\left|C_{i}\right| \geq 2$, for $1 \leq i \leq k$, and $e_{j}$ is an invariant for $1 \leq i \leq l$. Thus, we have $n=\left|C_{1}\right|+\left|C_{2}\right|+\cdots+\left|C_{k}\right|+l$.

An alternating group network $A N_{n}$ [27] is defined to be a Cayley graph $G=$ $G(V, E)$ on the alternating group $A_{n}$, where $V$ is the set of all even permutations of $\langle n\rangle=\{1,2, \ldots, n\}$ and $E$ consists of symmetric edges $(u, v)$ such that two permutations $u$ and $v$ are connected by an edge iff one can be reached from the other through the operations $v=f(u), f \in\left\{g_{l}, g_{r}, z_{i} \mid i=4, \ldots, n\right\}$. In the latter

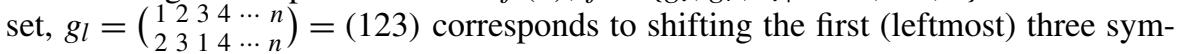
bols cyclically to the left by one position. Similarly, $g_{r}=\left(\begin{array}{llllll}1 & 2 & 3 & 4 & \cdots & n \\ 3 & 1 & 2 & 4 & \cdots & n\end{array}\right)=(312)$ implies shifting the first three symbols cyclically to the right by one position. Finally,

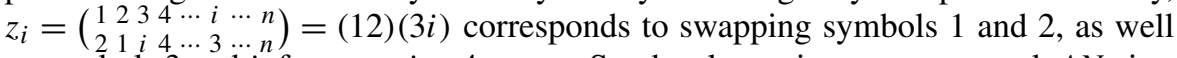
as symbols 3 and $i$, for some $i=4, \ldots, n$. So, the alternating group network $A N_{n}$ is a regular graph with $n ! / 2$ nodes, $n !(n-1) / 4$ edges, and node degree $n-1$. Youhu [27] has shown that $A N_{n}$ is Hamiltonian and has a diameter of $\lceil 3(n-2) / 2\rceil$. Each alternating group network $A N_{n}$ can be decomposed into $n$ sub-alternating group networks $A N_{n}^{1}, A N_{n}^{2}, \ldots, A N_{n}^{n}$, where each $A N_{n}^{i}$ fixes $i$ in the last position of the label strings representing the vertices and is isomorphic to $A N_{n-1}$. The edges that cross between these sub-alternating group networks constitute a perfect matching. 
Let $p=p_{1} p_{2} \ldots p_{n}$ be an even permutation representing one vertex of $A N_{n}$. The symbol $p_{i}$ in $p=p_{1} p_{2} \ldots p_{n}$ is fixed if $p_{i}=i$, and it is misplaced if $p_{i} \neq i$. The vertex $e=12 \ldots n$ is the identity vertex for which $p_{i}=i$ for all $1 \leq i \leq n$. In devising a routing algorithm, the vertex symmetry of $A N_{n}$ allows us to assume that $e$ is the destination vertex. We aim to construct $n-1$ vertex-disjoint paths from an arbitrary vertex $p$ to $e$ by "correcting" each non-fixed symbol to a fixed symbol. Similar to the corresponding operations for the star graph and the $(n, k)$-star, a non-fixed symbol should be moved to its desired position by first moving it to position 3. Non-fixed symbols can be presented within a cycle representation, and cyclically shifting the symbols in one cycle does not alter the occupying property of each symbol. Assume that the cycle representation for vertex $p$ is $C_{1} C_{2} \ldots C_{k}$ with $C_{i}=\left(r_{i, 1}, r_{i, 2}, \ldots, r_{i, k i}\right)$, where $k_{i}$ is the length of the cycle $C_{i}$. The symbol $r_{i, j}$ is the $j$ th symbol of $C_{i}$, and $r_{i, 1}$ is the head of $C_{i}$. In particular, if a cycle contains the symbol 3, we always assume the cycle is $C_{1}$, and normalize $C_{1}$ 's representation via rotations, so that the symbol 3 is the tail (last) symbol $r_{1, k 1}$ and $p_{3}$ is the head symbol $r_{1,1}$. Figure 1 depicts the first three alternating group networks $A N_{3}, A N_{4}$, and $A N_{5}$.

To prepare for the rest of our discussion, we reproduce in the following an optimal routing algorithm that can generate a shortest path between any two vertices of $A N_{n}$ [4]. Algorithm 1 is fully distributed, in the sense that it quickly determines the next node $p^{\prime}$ on a shortest path from the current node $p$ to the destination node $e$, using only the identities of $p$ and $e$.

Note that Algorithm 1 leads to the construction of a single shortest path from a source node to a destination node in $A N_{n}$, thus demonstrating that finding a shortest path for an alternating group network is relatively straightforward. We will see shortly, in Theorem 1 and its proof, that constructing the maximum number of parallel (node-disjoint) paths, all of which are close to minimum length, is a significantly more difficult endeavor.

The following result (Lemma 1) from reference [4] is needed for our subsequent discussion.

Lemma 1 [4] For any node $p$ of $A N_{n}$, let the canonical cycle structure be $C_{1} C_{2} \ldots C_{k}$, and define $m=\left|C_{1}\right|+\left|C_{2}\right|+\cdots+\left|C_{k}\right|$. If 3 is an invariant, then the distance $d(p, e)$ from node $p$ to the identity node $e$ is given by $h(p)$ defined below:

$$
\begin{aligned}
h(p) & =m+k & & \text { if } p_{1}=1 \text { and } p_{2}=2 \\
& =m+k-3 & & \text { if } p_{1}=2 \text { and } p_{2}=1 \\
& =m+k & & \text { if }\left|\left\{p_{1}, p_{2}\right\} \cap\{1,2\}\right|=1, \text { and } 1 \text { or } 2 \text { is an invariant } \\
& =m+k-1 & & \text { if }\left|\left\{p_{1}, p_{2}\right\} \cap\{1,2\}\right|=1, \text { and } 1,2 \text { belong to the same cycle } C_{i} \\
& =m+k & & \text { if }\left|\left\{p_{1}, p_{2}\right\} \cap\{1,2\}\right|=0, \text { and } 1,2 \text { belong to the same cycle } C_{i} \\
& =m+k-1 & & \text { if }\left|\left\{p_{1}, p_{2}\right\} \cap\{1,2\}\right|=0, \text { and } 1,2 \text { belong to different cycles }
\end{aligned}
$$

If 3 is not an invariant, then $d(p, e)=h(p)-2$.

\section{Construction of parallel paths}

In the following, we address the problem of constructing parallel paths between two arbitrary nodes of the alternating group network $A N_{n}$. The ideas for our construc- 


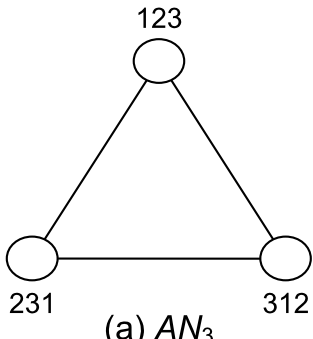

(a) $A N_{3}$
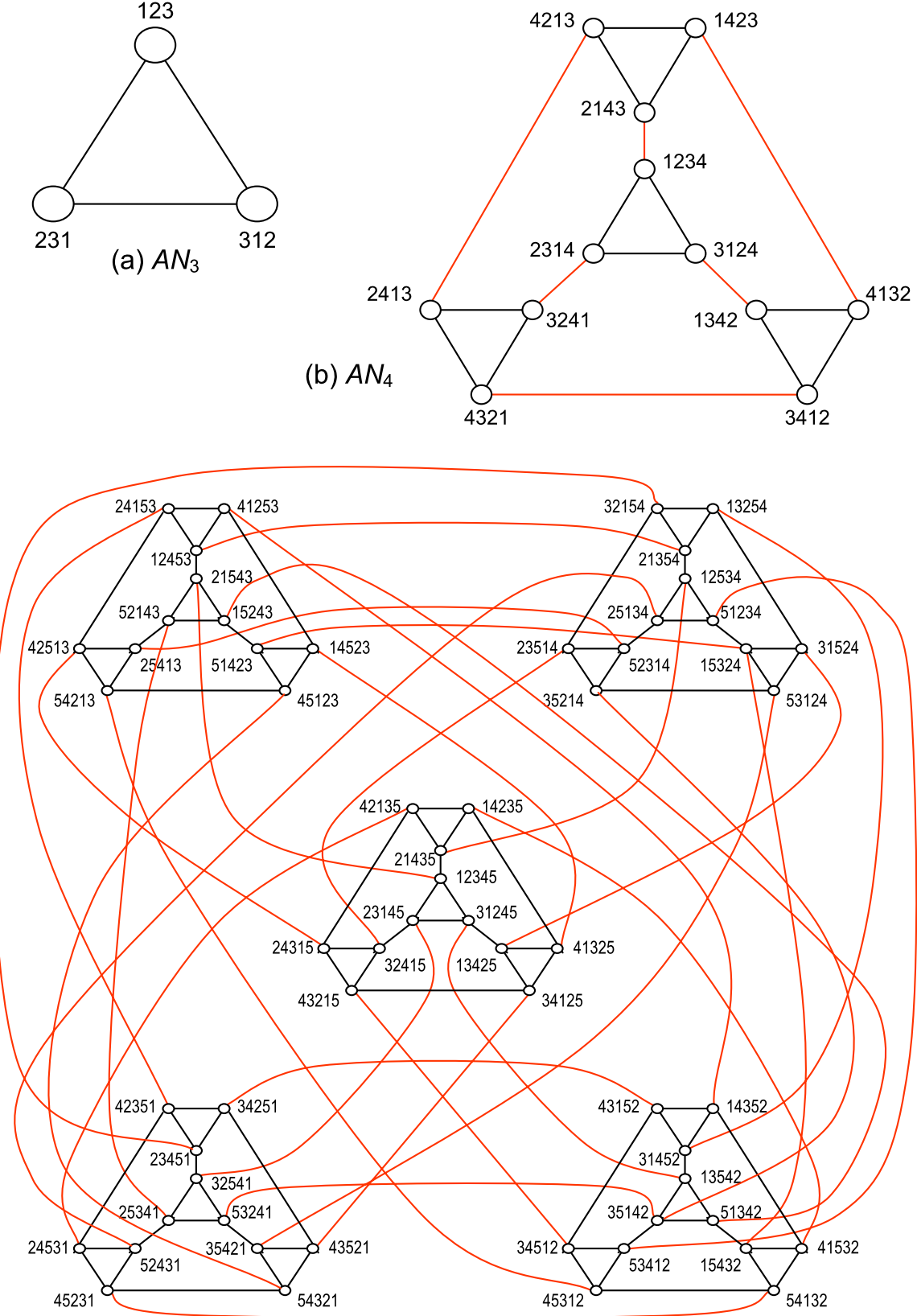

(c) $A N_{5}$

Fig. 1 The first three alternating group networks with 3, 12, and 60 nodes 
Algorithm 1: Route $\left(p=p_{1} p_{2} \cdots p_{n}\right)$

\{returns $p^{\prime}$, the first node on a shortest path from $p$ to $e$ in $A N_{n}$ \}

Case 1: if $p_{3}>3$

then $p^{\prime}=p z_{p_{3}}$

endif

Case 2: if $p_{3}=3$

then if $p_{4}=4, p_{5}=5, \ldots, p_{n}=n$

then stop

else $p^{\prime}=p z_{t}$, where $t>3$ and $p_{t} \neq t$

endif

endif

Case 3: if $p_{3}<3$

then if $p_{1}<3$ or $p_{2}<3$

then if $p_{1}<3$

then $p^{\prime}=p g_{r}$

else $p^{\prime}=p g_{l}$

endif

else if $\left(p_{3}=1\right.$ and 2 is not in the cycle $\left.(31 \cdots)\right)$ or

$\left(p_{3}=2\right.$ and 1 is not in the cycle $\left.(32 \cdots)\right)$

then $p^{\prime}=p g_{r}$

else $p^{\prime}=p g_{l}$

endif

endif

endif

tions originated from Day and Tripathi [6], and Lin and Duh [19]. We first construct a family of parallel paths of minimum distance, and then extend this family to its maximum possible size, $n-1$, by adding parallel paths that are only slightly longer than the shortest paths. We distinguish two cases. In the first case, the source and the destination permutations have the same third symbol, while in the second case, they have different third symbols. Because the alternating group network $A N_{n}$ is vertexsymmetric, we need only deal with the construction of parallel paths between an arbitrary node and the special node labeled with the identity permutation $e=12 \ldots n$.

Let $e_{j}$, where $1 \leq j \leq n-m$, be a fixed symbol in a vertex $p$, excluding the symbol 3. Note that we do not allow $e_{j}=3$, even when $p_{3}=3$ is fixed. An $e_{j}$-path is generated by first moving the symbol $e_{j}$ to position 3 , and then keeping $e_{j}$ away from its desired position, until all other symbols have been corrected. The $e_{j}$-path thus constructed is denoted by $\pi\left(e_{j}\right)$. Clearly, no $\pi\left(e_{j}\right)$ path can be constructed if $m=n$. We use underlining at each step to indicate which symbol is being corrected. Some steps in these paths do not correspond to a symbol correction, but to a preparation for a symbol correction by moving the desired symbol to the third position.

Theorem 1 There are $n-1$ vertex-disjoint paths between any two vertices of the alternating group network $A N_{n}$. Furthermore, the length of each of these paths is bounded by $d(u, v) \leq l \leq d(u, v)+4$, where $d(u, v)$ is the distance between $u$ and $v$. 
Table 1 The structure of the proof of Theorem 1 in terms of parts (A/B), cases (1-6), and subcases (a/b). The label given in the leftmost column corresponds to the (sub)section in Appendix A or B where the corresponding proof can be found

\begin{tabular}{|c|c|c|c|c|}
\hline Case & $p_{3}$ & $p_{1}, p_{2}$ & Status of 1 and 2 & Status of 3 when $p_{3} \neq 3$ \\
\hline A1 & $p_{3}=3$ & $\left\{p_{1}, p_{2}\right\}=\{1,2\}$ & $p_{1}=1$ and $p_{2}=2$ & \\
\hline A2 & & & $p_{1}=2$ and $p_{2}=1$ & \\
\hline A3 & & $\left\{p_{1}, p_{2}\right\} \cap\{1,2\}=\{r\}$ & $p_{1}=1$ or $p_{2}=2$ & \\
\hline A4 & & & 1,2 in same cycle & \\
\hline A5 & & & 1,2 in different cycles & \\
\hline A6 & & $\left\{p_{1}, p_{2}\right\} \cap\{1,2\}=\Phi$ & 1,2 in same cycle & \\
\hline B1 & $p_{3} \neq 3$ & $\left\{p_{1}, p_{2}\right\}=\{1,2\}$ & $p_{1}=1$ and $p_{2}=2$ & (no subcase used) \\
\hline B2 & & & $p_{1}=2$ and $p_{2}=1$ & \\
\hline B3a & & $\left\{p_{1}, p_{2}\right\} \cap\{1,2\}=\{r\}$ & $p_{1}=1$ or $p_{2}=2$ & $3, r$ in same cycle \\
\hline B3b & & & & $3, r$ in different cycles \\
\hline B4a & & & 1,2 in same cycle & 3 in same cycle as 1,2 \\
\hline B4b & & & & 3 in different cycle from 1,2 \\
\hline B5a & & & 1,2 in different cycles & 3 in same cycle as 1 or 2 \\
\hline $\mathrm{B} 5 \mathrm{~b}$ & & & & 3 in different cycle from 1,2 \\
\hline B6a & & $\left\{p_{1}, p_{2}\right\} \cap\{1,2\}=\Phi$ & 1,2 in same cycle & 3 in same cycle as 1,2 \\
\hline B6b & & & & 3 in different cycle from 1,2 \\
\hline
\end{tabular}

Proof By the vertex symmetry of $A N_{n}$, it suffices to show the result for one vertex labeled with an arbitrary even permutation $p=C_{1} C_{2} \ldots C_{k} e_{1} e_{2} \ldots e_{l}$ and the special vertex labeled with the identity permutation $e=12 \ldots n$. The proof is composed of two parts, each with several cases. Because of the many cases and tedious derivations involved, the proof is given in Appendices A (the case of $p_{3}=3$ ) and B (the case of $p_{3} \neq 3$ ). Table 1 lists the various cases and subcases in the proof for ready reference and to illustrate the proof outline.

Theorem 2 The family of $n-1$ paths from any vertex to the identity vertex e constructed by the parallel routing rule above are internode-disjoint, meaning that they do not share any vertex other than their endpoints.

Proof We only show that the family of $n-1$ paths from any vertex $p$ with $p_{3} \neq 3$ to $e$ are node-disjoint. The proof of the case with $p_{3}=3$ is similar and is thus omitted for brevity.

(1) Let $\pi\left(r_{i, 1}\right)$ denote the path constructed from $p$ to $e$ along which the $m$ misplaced symbols are corrected according to the order $\left(C_{i}, C_{i+1}, \ldots, C_{k}, C_{1}, C_{2}, \ldots\right.$, $\left.C_{i-1}\right)$. Similarly, let $\pi\left(r_{j, 1}\right)$ be the path constructed from $p$ to $e$ along which the $m$ misplaced symbols are corrected according to the order $\left(C_{j}, C_{j+1}, \ldots, C_{k}, C_{1}\right.$, $\left.C_{2}, \ldots, C_{j-1}\right)$, where $i<j$. Let $\pi_{t}\left(r_{i, 1}\right)$ be the $t$ th vertex in the path $\pi\left(r_{i, 1}\right)$, where, $\pi_{0}\left(r_{i, 1}\right)=p$. Obviously, $\pi_{1}\left(r_{i, 1}\right)$ is different from every vertex in $\pi\left(r_{j, 1}\right)$, because $\pi_{1}\left(r_{i, 1}\right)$ and $\pi_{1}\left(r_{j, 1}\right)$ are distinct neighbors of $p$ and a symbol $r_{j, 1}$ in $\pi_{1}\left(r_{j, 1}\right)$, with the exception of $p$, has already been corrected to its desired po- 
sition. Each vertex $\pi_{t}\left(r_{i, 1}\right), t \geq 2$, has the symbol $r_{i, 1}$ fixed, but $r_{i-1, k i-1}$ misplaced. By the rotation property, the correction order of $r_{i-1, k i-1}$ precedes that of $r_{i, 1}$ in the path $\pi\left(r_{j, 1}\right)$. So, there are no vertices in $\pi\left(r_{j, 1}\right)$ that have the symbol $r_{i, 1}$ fixed, but $r_{i-1, k i-1}$ misplaced. Therefore, $\pi\left(r_{i, 1}\right)$ and $\pi\left(r_{j, 1}\right)$ are disjoint.

(2) For each $r_{i, j} \neq r_{i, 1}\left(2 \leq i \leq k, 2 \leq j \leq k_{i}\right)$, the path $\pi\left(r_{i, j}\right)$ is constructed from $p$ to $e$ along which the $m$ misplaced symbols are corrected according to the or$\operatorname{der}\left(r_{i, j}, r_{i, j+1}, \ldots, r_{i, k i}, C_{i+1}, \ldots, C_{k}, r_{1, k 1}, r_{1,1}, r_{1,2}, \ldots, r_{1, k 1-1}, C_{2}, \ldots, C_{i-1}\right.$, $\left.r_{i, 1}, r_{i, 2}, \ldots, r_{i, j-1}\right)$. The fact that the paths constructed by this rule are disjoint from each other can be proven by a method similar to that in paragraph (1) above. Because the correction order of the element $r_{i, j-1}$ precedes that of $r_{i, j}$ in the paths constructed in paragraph (1) but not in (2), the two sets of paths under (1) and (2) must be disjoint.

(3) For each $r_{i, j} \neq r_{i, 1}\left(i=1, k_{1} \geq 3,2 \leq j \leq k_{1}\right), \pi\left(r_{1, j}\right)$ with $3 \leq j \leq k_{1}-1$ is constructed along which $m$ misplaced symbols are corrected according to the or$\operatorname{der}\left(r_{1, j}, r_{1, j+1}, \ldots, r_{1, k 1}, C_{2}, \ldots, C_{k}, r_{1,1}, r_{1,2}, \ldots, r_{1, j-1}\right)$. Similarly, $\pi\left(r_{1, k 1}\right)$ with $k_{1} \geq 3$ is constructed along which $m$ misplaced symbols are corrected according to the order $\left(r_{1, k 1}, C_{2}, \ldots, C_{k}, r_{1,2}, \ldots, r_{1, k 1-1}, r_{1,1}\right)$. Arguments similar to those under paragraphs (1) and (2) establish that the paths constructed in (3) are disjoint from each other and from those constructed earlier.

(4) The paths $\pi\left(e_{j}\right)$ are obtained by first diverting one fixed symbol $e_{j}$, other than 3 , by moving it from its correct position to the third position, then along the correction order sequence $\left(C_{1}, C_{2}, \ldots, C_{k}\right)$. Finally, the diverted symbol $e_{j}$ is returned to its desired position. Such a path $\pi\left(e_{j}\right)$ is node-disjoint from any path $\pi\left(r_{i, j}\right)$ constructed in paragraphs (1)-(3), because the symbol $e_{j}$ is misplaced all along $\pi\left(e_{j}\right)$, while $e_{j}$ is in its desired position in $\pi\left(r_{i, j}\right)$. On the other hand, the two paths obtained by diverting $e_{i}$ and $e_{j}\left(e_{i} \neq e_{j}\right)$, respectively, are nodedisjoint because $e_{j}$ is misplaced all along the path $\pi\left(e_{i}\right)$ but fixed all along the path $\pi\left(e_{j}\right)$, while $e_{j}$ is misplaced along the path $\pi\left(e_{j}\right)$ but fixed in the path $\pi\left(e_{i}\right)$.

Lemma 2 [19] If $G$ is a regular graph with connectivity $\kappa \geq 2$, then $d_{\kappa}(G) \geq$ $D(G)+1$, where $D(G)$ is the diameter of $G$.

Theorem 3 The wide diameter $d_{n-1}\left(A N_{n}\right)$ of $A N_{n}$ is bounded as $D\left(A N_{n}\right)+1 \leq$ $d_{n-1}\left(A N_{n}\right) \leq D\left(A N_{n}\right)+2$, which means that it is within one unit of the smallest possible.

Proof By Lemma 2, we only need to show that $d_{n-1}\left(A N_{n}\right) \leq D\left(A N_{n}\right)+2$. For convenience, we use $d_{n-1}(p, e)$ to denote the length of the longest of the $n-1$ paths constructed in the proof of Theorem 1 . We limit our proof to a single case, A1, where $d_{n-1}(p, e)=m+k+2=n-l+k+2$, with $l \geq 3$. Other cases can be dealt with similarly. Note that $\lceil 3(n-2) / 2\rceil$ equals the diameter $D\left(A N_{n}\right)$ of $A N_{n}$.

(1) If $n-l=0 \bmod 4$, that is, $(n-l) / 2$ is an even integer, then $k \leq(n-l) / 2$ and $d_{n-1}(p, e)=n-l+k+2 \leq 3(n-l) / 2+2 \leq 3(n-3) / 2+2=3(n-2) / 2+$ $1 / 2 \leq\lceil 3(n-2) / 2\rceil+1 / 2$. 
(2) If $n-l=2 \bmod 4$, that is, $(n-l) / 2$ is an odd integer, then $k \leq(n-l) / 2-1$ and $d_{n-1}(p, e)=n-l+k+2 \leq 3(n-l) / 2+1 \leq 3(n-3) / 2+1=3(n-2) / 2-$ $1 / 2 \leq\lceil 3(n-2) / 2\rceil-1 / 2$.

(3) If $n-l=1 \bmod 4$, that is, $(n-l-1) / 2$ is an even integer, then $k \leq(n-l-1) / 2$ and $d_{n-1}(p, e)=n-l+k+2 \leq 3(n-l) / 2+1 / 2 \leq 3(n-3) / 2+1 / 2=3(n-$ 2) $/ 2-1 \leq\lceil 3(n-2) / 2\rceil-1$.

(4) If $n-l=3 \bmod 4$, that is, $(n-l-1) / 2$ is an odd integer, then $k \leq(n-l-1) / 2$ and $d_{n-1}(p, e)=n-l+k+2 \leq 3(n-l) / 2+3 / 2 \leq 3(n-3) / 2+3 / 2=3(n-$ 2) $/ 2 \leq\lceil 3(n-2) / 2\rceil$.

The results of paragraphs (1)-(4) above imply that $d_{n-1}(p, e) \leq D\left(A N_{n}\right)+2$.

\section{Conclusion}

The node-to-node internally disjoint paths problem is to find $k$ paths between two arbitrary nodes $u$ and $v$ in a $k$-connected graph such that these paths do not share any nodes other than their two endpoints. The existence of such paths is an indication of the robustness of an interconnection network, in the sense of it being maximally fault tolerant, given that (by definition) a $k$-connected graph must contain some pairs of nodes that are not connected by more than $k$ parallel paths. Once such paths are obtained, they enable fault-tolerant routing by using alternate paths when a particular path is unavailable. Similarly, high-throughput routing becomes possible by using parallel paths to send multiple pieces of long messages at once.

Alternating group networks are not the only networks to be maximally fault tolerant in the sense defined above. However, in general, some of the $k$ parallel paths between nodes $u$ and $v$ may be significantly longer than the minimum distance $d(u, v)$. If, in a particular network, the $k$ parallel paths can be constructed so that their length is bounded by $d(u, v)+\varepsilon$, for some small constant $\varepsilon$, then the network is not only highly robust, but also can maintain its high performance in the presence of multiple faulty nodes and links. We have shown $A N_{n}$ to be such a network and established that $\varepsilon \leq 4$. Of course, we are assuming that faults are readily detectable, so that message transmission over the associated paths can be avoided by means of suitably updating routing tables or other routing mechanisms. This is not an unreasonable assumption, given the use of error-detecting codes and other concurrent monitoring schemes.

A useful variation on the node-to-node internally disjoint paths problem is the node-to-set disjoint paths problem, defined as follows. Given a node $u$ and $k$ other nodes $w_{1}, w_{2}, \ldots, w_{k}$, find $k$ node-disjoint paths that connect node $u$ to the nodes $w_{i}, 1 \leq i \leq k$. Our ongoing research is centered on this problem. A solution to the node-to-set disjoint paths problem would be quite helpful in broadcasting or multicasting. A set of desired destination nodes can be reached by choosing $k$ intermediate nodes, sending messages to those nodes via disjoint paths, and recursively spreading the message from the newly informed $k$ sources.

Acknowledgements This work was supported in part by the Natural Science Foundation of Fujian Province through grants 2007F3025 and 2007J0316. 
Open Access This article is distributed under the terms of the Creative Commons Attribution Noncommercial License which permits any noncommercial use, distribution, and reproduction in any medium, provided the original author(s) and source are credited.

\section{Appendix A: Proof of Theorem 1 for $p_{3}=3$}

Please refer to Sect. 3 of the paper, Table 1 in particular, for the outline and structure of the proof.

A.1 The case of $p_{1}=1$ and $p_{2}=2$

(1) For each $r_{i, j}\left(1 \leq i \leq k, 1 \leq j \leq k_{i}\right)$ in the cycle representation of $p$, a path $\pi\left(r_{i, j}\right)$ of minimum length $m+k$ is constructed from $p$ to $e$ along which $m$ misplaced symbols are corrected in order of the sequence $\left(r_{i, j}, r_{i, j+1}, \ldots, r_{i, k i}\right.$, $\left.C_{i+1}, \ldots, C_{k}, C_{1}, C_{2}, \ldots, C_{i-1}, r_{i, 1}, r_{i, 2}, \ldots, r_{i, j-1}\right)$. Since the $m$ misplaced elements each can be seen as the first element of the correction sequence by a suitable number of left circular shifts, we have $m$ vertex-disjoint paths, each of length $m+k$. For example, given $p=12367458=(46)(57)(1)(2)(3)(8)$ in $A N_{8}$, we construct these optimal parallel paths:

$$
\begin{aligned}
& \pi_{1}=12367458 \rightarrow 21 \underline{467358} \rightarrow 12 \underline{6} 47358 \rightarrow 21347658 \rightarrow 12 \underline{5} 47638 \\
& \rightarrow 21 \underline{7} 45638 \rightarrow e \\
& \pi_{2}=12367458 \rightarrow 21 \underline{6} 37458 \rightarrow 12437658 \rightarrow 21 \underline{5} 37648 \rightarrow 12 \underline{73} 35648 \\
& \rightarrow 21 \underline{4} 35678 \rightarrow e \\
& \pi_{3}=12367458 \rightarrow 21 \underline{5} 67438 \rightarrow 12 \underline{765438} \rightarrow 21365478 \rightarrow 12 \underline{4} 65378 \\
& \rightarrow 21 \underline{6} 45378 \rightarrow e \\
& \pi_{4}=12367458 \rightarrow 21 \underline{7} 63458 \rightarrow 12563478 \rightarrow 21 \underline{463578} \rightarrow 12 \underline{6} 43578 \\
& \rightarrow 21 \underline{543678 \rightarrow e}
\end{aligned}
$$

(2) An $e_{j}$-path is generated by first moving one fixed $e_{j}$ to position 3 , and then keeping $e_{j}$ away from its desired position until the other symbols have been corrected in order of the sequence $\left(C_{1}, C_{2}, \ldots, C_{k}\right)$. The $j$ th $e_{j}$-path in this set, $\pi\left(e_{j}\right)$, is of length $m+k+2$ and is constructed in order of the correction sequence $\left(e_{j}, C_{1}, C_{2}, \ldots, C_{k}, e_{j}\right)$.

$$
\begin{aligned}
& \pi_{5}=12367458 \rightarrow 23 \underline{1} 67458 \rightarrow 32 \underline{4} 67158 \rightarrow 23 \underline{6} \underline{4} 4158 \rightarrow 32147658 \\
& \rightarrow 23 \underline{5} 47618 \rightarrow 32 \underline{7} 45618 \rightarrow 23 \underline{1} 45678 \rightarrow e \\
& \pi_{6}=12367458 \rightarrow 31 \underline{2} 67458 \rightarrow 13 \underline{4} 67258 \rightarrow 31 \underline{6} 47258 \rightarrow 13247658 \\
& \rightarrow 31 \underline{5} 47628 \rightarrow 13 \underline{7} 45628 \rightarrow 31 \underline{2} 45678 \rightarrow e \\
& \pi_{7}=12367458 \rightarrow 21 \underline{867453} \rightarrow 12 \underline{467853} \rightarrow 21 \underline{6} 47853 \rightarrow 12847653 \\
& \rightarrow 21 \underline{5} 47683 \rightarrow 12 \underline{7} 45683 \rightarrow 21 \underline{8} 45673 \rightarrow e
\end{aligned}
$$


A.2 The case of $p_{1}=2$ and $p_{2}=1$

Since the elements of (12) are placed at their desired positions only at the end of the correction process, that is to say, (12) automatically gets sorted when all the non-fixed symbols have been sorted, we may always set $C_{k}=(12)$.

(1) For each $r_{i, j}\left(1 \leq i \leq k, 1 \leq j \leq k_{i}\right)$ in the cycle representation of $p$, a path $\pi\left(r_{i, j}\right)$ of minimum length $m+k-3$ is constructed from $p$ to $e$ along which $m-2$ misplaced symbols, excluding the elements of $C_{k}=(12)$, are corrected in order of the sequence $\left(r_{i, j}, r_{i, j+1}, \ldots, r_{i, k i}, C_{i+1}, \ldots, C_{k-1}, C_{1}, C_{2}, \ldots, C_{i-1}\right.$, $\left.r_{i, 1}, r_{i, 2}, \ldots, r_{i, j-1}\right)$. Since the $m-2$ misplaced elements each can be seen as the first element of the correction sequence by a suitable number of left circular shifts, we have $m-2$ disjoint paths each of length $m-2+k-1=m+k-3$. For example, given $p=213749586=(475)(69)(21)(3)(8)$ in $A N_{9}$, we construct these parallel paths:

$$
\begin{aligned}
\pi_{1}= & 213749586 \rightarrow 12 \underline{4} 739586 \rightarrow 21 \underline{7} 439586 \rightarrow 12 \underline{5} 439786 \rightarrow 213459786 \\
& \rightarrow 12 \underline{6} 459783 \rightarrow 21 \underline{9} 456783 \rightarrow e \\
\pi_{2}= & 213749586 \rightarrow 12 \underline{7} 349586 \rightarrow 21 \underline{5} 349786 \rightarrow 124359786 \rightarrow 21 \underline{6} 359784 \\
& \rightarrow 12 \underline{9} 356784 \rightarrow 21 \underline{4356789} \rightarrow e \\
\pi_{3}= & 213749586 \rightarrow 12 \underline{5} 749386 \rightarrow 214759386 \rightarrow 12 \underline{6} 759384 \rightarrow 21 \underline{9756384} \\
& \rightarrow 12 \underline{4} 756389 \rightarrow 21 \underline{7} 456389 \rightarrow e \\
\pi_{4}= & 213749586 \rightarrow 12 \underline{6} 749583 \rightarrow 21 \underline{9} 746583 \rightarrow 123746589 \rightarrow 21 \underline{47} 36589 \\
& \rightarrow 12 \underline{7} 436589 \rightarrow 21 \underline{5} 436789 \rightarrow e \\
\pi_{5}= & 213749586 \rightarrow 12 \underline{9} 743586 \rightarrow 216743589 \rightarrow 12 \underline{4763589} \rightarrow 21 \underline{74} 463589 \\
& \rightarrow 12 \underline{5} 463789 \rightarrow 21 \underline{6} 453789 \rightarrow e
\end{aligned}
$$

(2) An $e_{j}$-path is generated by first moving one fixed symbol $e_{j}$ to position 3 , and then keeping $e_{j}$ away from its desired position until the other symbols have been corrected according to the order of the sequence $\left(C_{1}, C_{2}, \ldots, C_{k-1}\right)$. The $j$ th $e_{j}$-path in this set, $\pi\left(e_{j}\right)$, is of length $m-2+k-1+2=m+$ $k-1$ and is constructed according to the order of the correction sequence $\left(e_{j}, C_{1}, C_{2}, \ldots, C_{k-1}, e_{j}\right)$, where $e_{j}$ can be an element of $C_{k}=(12)$.

$$
\begin{aligned}
\pi_{6}= & 213749586 \rightarrow 32 \underline{17} 49586 \rightarrow 23 \underline{4} 719586 \rightarrow 32 \underline{7} 419586 \rightarrow 23 \underline{5} 419786 \\
& \rightarrow 321459786 \rightarrow 23 \underline{6} 459781 \rightarrow 32 \underline{9} 456781 \rightarrow 23 \underline{1} 456789 \rightarrow e \\
\pi_{7}= & 213749586 \rightarrow 13 \underline{2} 749586 \rightarrow 31 \underline{4729586} \rightarrow 13 \underline{7} 429586 \rightarrow 31 \underline{5} 429786 \\
& \rightarrow 132459786 \rightarrow 31 \underline{6} 459782 \rightarrow 13 \underline{9} 456782 \rightarrow 31 \underline{2} 456789 \rightarrow e \\
\pi_{8}= & 213749586 \rightarrow 12 \underline{8} 749536 \rightarrow 21 \underline{4} 789536 \rightarrow 12 \underline{7} 489536 \rightarrow 21 \underline{5} 489736 \\
& \rightarrow 128459736 \rightarrow 21 \underline{6} 459738 \rightarrow 12 \underline{9} 456738 \rightarrow 21 \underline{8} 456739 \rightarrow e
\end{aligned}
$$


A.3 The case of $\left\{p_{1}, p_{2}\right\} \cap\{1,2\}=\{r\}$, with $p_{1}=1$ or $p_{2}=2$

The construction in this case is similar to that of Sect. A.1.

A.4 The case of $\left\{p_{1}, p_{2}\right\} \cap\{1,2\}=\{r\}$, with 1 and 2 in the same cycle $C_{i}$

(1) For each $r_{i, j}\left(1 \leq i \leq k, 1 \leq j \leq k_{i}\right)$ in the cycle representation of $p$, a path $\pi\left(r_{i, j}\right)$ of minimum length $m+k-1$ is constructed from $p$ to $e$ along which $m$ misplaced symbols are corrected in order of the sequence $\left(r_{i, j}, r_{i, j+1}, \ldots, r_{i, k i}, C_{i+1}, \ldots, C_{k}, C_{1}, C_{2}, \ldots, C_{i-1}, r_{i, 1}, r_{i, 2}, \ldots, r_{i, j-1}\right)$.

Since $m$ misplaced elements each can be seen as the first element of the correction sequence by a suitable number of left circular shifts, we have $m$ disjoint paths each of length $m+k-1$. For example, given $p=27356418=(712)(456)(3)(8)$ in $A N_{8}$, we construct these parallel paths:

$$
\begin{aligned}
\pi_{1}= & 27356418 \rightarrow 32 \underline{756418} \rightarrow 23156478 \rightarrow 12356478 \rightarrow 21 \underline{4} 56378 \\
& \rightarrow 12 \underline{5} 46378 \rightarrow 21 \underline{6} 45378 \rightarrow e \\
\pi_{2}= & 27356418 \rightarrow 72156438 \rightarrow 21756438 \rightarrow 12 \underline{4} 56738 \rightarrow 21 \underline{5} 46738 \\
& \rightarrow 12 \underline{6} 45738 \rightarrow 21 \underline{74} 55638 \rightarrow e \\
\pi_{3}= & 27356418 \rightarrow 72 \underline{4} 56318 \rightarrow 27 \underline{5} 46318 \rightarrow 72 \underline{6} \underline{4} 5318 \rightarrow 27345618 \\
& \rightarrow 32 \underline{7} 45618 \rightarrow 23145678 \rightarrow e \\
\pi_{4}= & 27356418 \rightarrow 72 \underline{5} 36418 \rightarrow 27 \underline{6} 35418 \rightarrow 72435618 \rightarrow 24 \underline{7} 35618 \\
& \rightarrow 42 \underline{1} 35678 \rightarrow 21 \underline{4} 35678 \rightarrow e \\
\pi_{5}= & 27356418 \rightarrow 72 \underline{6} 36418 \rightarrow 27453618 \rightarrow 42 \underline{753618} \rightarrow 24153678 \\
& \rightarrow 12 \underline{4} 53678 \rightarrow 21 \underline{5} 43678 \rightarrow e
\end{aligned}
$$

(2) An $e_{j}$-path is generated by first moving one fixed symbol $e_{j}$ (where $e_{j}=2$ is possible) to position 3 , and then keeping $e_{j}$ away from its desired position until the other symbols have been corrected. The $j$ th $e_{j}$-path in this set, $\pi\left(e_{j}\right)$, is of length $m+k+1$ and is constructed in the order of the correction sequence $\left(e_{j}, C_{1}, C_{2}, \ldots, C_{k-1}, e_{j}\right)$.

$$
\begin{aligned}
\pi_{6}= & 27356418 \rightarrow 73 \underline{2} 56418 \rightarrow 37156428 \rightarrow 13 \underline{75} 6428 \rightarrow 31 \underline{4} 56728 \\
& \rightarrow 13 \underline{5} 46728 \rightarrow 31 \underline{6} 45728 \rightarrow 13745628 \rightarrow 31 \underline{2} 45678 \rightarrow e \\
\pi_{7}= & 27356418 \rightarrow 72 \underline{8} 56413 \rightarrow 27156483 \rightarrow 12 \underline{7} 56483 \rightarrow 21 \underline{4} 56783 \\
& \rightarrow 12 \underline{5} 46783 \rightarrow 21 \underline{6} 45783 \rightarrow 12745683 \rightarrow 21 \underline{8} 45673 \rightarrow e
\end{aligned}
$$

A.5 The case of $\left\{p_{1}, p_{2}\right\} \cap\{1,2\}=\{r\}$, with 1 and 2 in different cycles

The construction in this case is similar to that of Sect. A.4. 
A.6 The case of $\left\{p_{1}, p_{2}\right\} \cap\{1,2\}=\Phi$, with 1 and 2 in the same cycle $C_{i}$

The construction in this case is similar to that of Sect. A.1.

This concludes the first part of the proof of Theorem 1, corresponding to the condition $p_{3}=3$. The second part of the proof, for $p_{3} \neq 3$, is presented in Appendix B.

\section{Appendix B: Proof of Theorem 1 for $p_{3} \neq 3$}

Please refer to Sect. 3 of the paper, Table 1 in particular, for the outline and structure of the proof.

B.1 The case of $p_{1}=1$ and $p_{2}=2$

(1) For each head in the cycle representation of $p$, that is, $r_{i, 1}(1 \leq i \leq k)$, a path $\pi\left(r_{i, j}\right)$ is constructed from $p$ to $e$ along which $m$ misplaced symbols are corrected in order of the sequence $\left(C_{i}, C_{i+1}, \ldots, C_{k}, C_{1}, C_{2}, \ldots, C_{i-1}\right)$, where this order is obtained by performing on $\left(C_{1}, C_{2}, \ldots, C_{k}\right)$ a number of left circular shifts which make the head of the cycle $C_{i}$ become the first element of the correction sequence. So, we have $k$ paths of minimum length $m+k-2$. For example, given $p=125463978=(563)(798)(1)(2)(4)$ in $A N_{9}$, we construct two optimal parallel paths as follows:

$$
\begin{aligned}
\pi_{1}= & 12 \underline{5} 463978 \rightarrow 21 \underline{6} 453978 \rightarrow 123456978 \rightarrow 21 \underline{7456938} \rightarrow 12 \underline{9} 456738 \\
& \rightarrow 21 \underline{8} 456739 \rightarrow e \\
\pi_{2}= & 125463978 \rightarrow 21 \underline{7} 463958 \rightarrow 12 \underline{9} 463758 \rightarrow 21 \underline{8} 463759 \rightarrow 12 \underline{5} 463789 \\
& \rightarrow 21 \underline{6} 453789 \rightarrow e
\end{aligned}
$$

(2) For each $r_{i, j} \neq r_{i, 1}\left(2 \leq i \leq k, 2 \leq j \leq k_{i}\right)$ in the cycle representation of $p$, a path $\pi\left(r_{i, j}\right)$ is built from $p$ to $e$ along which $m$ misplaced symbols are corrected in order of the sequence $\left(r_{i, j}, r_{i, j+1}, \ldots, r_{i, k i}, C_{i+1}, \ldots, C_{k}, r_{1, k 1}, r_{1,1}, r_{1,2}, \ldots\right.$, $\left.r_{1, k 1-1}, C_{2}, \ldots, C_{i-1}, r_{i, 1}, r_{i, 2}, \ldots, r_{i, j-1}\right)$. So we have $m-(k-1)-\left|C_{1}\right|$ paths, each of length $m+k$. Continuing with our example, we have:

$$
\begin{aligned}
\pi_{3}= & 125463978 \rightarrow 21 \underline{9} 463578 \rightarrow 12 \underline{8} 463579 \rightarrow 217463589 \rightarrow 123467589 \\
& \rightarrow 21 \underline{5} 467389 \rightarrow 12 \underline{6} 457389 \rightarrow 21 \underline{7} 456389 \rightarrow e \\
\pi_{4}= & 125463978 \rightarrow 21 \underline{8} 463975 \rightarrow 127463985 \rightarrow 213467985 \rightarrow 12 \underline{5} 467983 \\
& \rightarrow 21 \underline{6} \underline{4} 457983 \rightarrow 12 \underline{7} 456983 \rightarrow 21 \underline{9} 456783 \rightarrow e
\end{aligned}
$$

(3) For each $r_{i, j} \neq r_{i, 1}\left(i=1,1 \leq j \leq k_{1}\right)$ in the cycle representation of $p$, a path $\pi\left(r_{i, j}\right)$ is constructed as follows; note that the path $\pi\left(r_{1,1}\right)$ is the same as the path $\pi\left(r_{1,2}\right)$ :

Each path $\pi\left(r_{1, j}\right)$, with $k_{1} \geq 3$, is constructed along which $m$ misplaced symbols are corrected in order of the sequence $\left(r_{1, j}, r_{1, j+1}, \ldots, r_{1, k 1}, C_{2}, \ldots, C_{k}\right.$, $\left.r_{1,1}, r_{1,2}, \ldots, r_{1, j-1}\right)$. 
Each path $\pi\left(r_{1, k 1}\right)$ is constructed along which $m$ misplaced symbols are corrected according to the order of the sequence $\left(r_{1, k 1}, C_{2}, \ldots, C_{k}, r_{1,2}, \ldots\right.$, $\left.r_{1, k 1-1}, r_{1,1}\right)$.

So we have $\left|C_{1}\right|-2$ paths, each of length $m+k$. Continuing with our example, we get:

$$
\begin{aligned}
\pi_{5}= & 125463978 \rightarrow 213465978 \rightarrow 12 \underline{7} 465938 \rightarrow 21 \underline{9} 465738 \rightarrow 12 \underline{8} 465739 \\
& \rightarrow 213465789 \rightarrow 12 \underline{6} 45789 \rightarrow 21 \underline{5} 436789 \rightarrow e
\end{aligned}
$$

(4) An $e_{j}$-path is generated by first moving a fixed element $e_{j}$ to position 3 , and then keeping $e_{j}$ away from its desired position until the other symbols have been corrected. The $j$ th $e_{j}$-path, $\pi\left(e_{j}\right)$, is constructed in order of the correction sequence $\left(e_{j}, C_{2}, \ldots, C_{k}, r_{1, k 1}, r_{1,1}, r_{1,2}, \ldots, r_{i, k 1-1}, e_{j}\right)$. This process yields $n-m$ paths, each of length $m+k+2$. For our example, the paths are:

$$
\begin{aligned}
\pi_{6}= & 125463978 \rightarrow 25 \underline{1} 463978 \rightarrow 52 \underline{7} 463918 \rightarrow 25 \underline{9} 463718 \rightarrow 52 \underline{8} 463719 \\
& \rightarrow 251463789 \rightarrow 523461789 \rightarrow 23 \underline{5} 461789 \rightarrow 32 \underline{6} 451789 \\
& \rightarrow 23 \underline{1} 456789 \rightarrow e \\
\pi_{7}= & 125463978 \rightarrow 51 \underline{2} 463978 \rightarrow 15 \underline{7} 463928 \rightarrow 51 \underline{9} 463728 \rightarrow 15 \underline{8} 463729 \\
& \rightarrow 512463789 \rightarrow 153462789 \rightarrow 31 \underline{5} 462789 \rightarrow 13 \underline{6} 452789 \\
& \rightarrow 31 \underline{2} 456789 \rightarrow e \\
\pi_{8}= & 125463978 \rightarrow 21 \underline{4} 563978 \rightarrow 12 \underline{7} 563948 \rightarrow 21 \underline{9} 563748 \rightarrow 12 \underline{8} 563749 \\
& \rightarrow 214563789 \rightarrow 123564789 \rightarrow 21 \underline{5} 364789 \rightarrow 12 \underline{6} 354789 \\
& \rightarrow 21 \underline{4} 356789 \rightarrow e
\end{aligned}
$$

B.2 The case of $p_{1}=2$ and $p_{2}=1$

(1) For each head in the cycle representation of $p$, that is, $r_{i, 1}(1 \leq i \leq k-1)$, a path $\pi\left(r_{i, 1}\right)$ is constructed from $p$ to $e$ along which $m-2$ misplaced symbols, excluding elements of $C_{k}=(12)$, are corrected in order of the sequence $\left(C_{i}, C_{i+1}, \ldots, C_{k-1}, C_{1}, C_{2}, \ldots, C_{i-1}\right)$, where the order is obtained by performing on $\left(C_{1}, C_{2}, \ldots, C_{k-1}\right)$ a number of left circular shifts which make the head of the cycle $C_{i}$ become the first element of the correction sequence. This process yields $k-1$ paths of optimal length $m-2-(k-1)-2=m+k-5$. For example, given $p=21583476=(53)(486)(12)(7)$ in $A N_{8}$, we construct the following $k-1$ parallel paths:

$$
\begin{aligned}
& \pi_{1}=21 \underline{583476} \rightarrow 12385476 \rightarrow 21 \underline{485376} \rightarrow 12 \underline{8} 45376 \rightarrow 21 \underline{6} 45378 \rightarrow e \\
& \pi_{2}=21583476 \rightarrow 12 \underline{4} 83576 \rightarrow 21 \underline{8} 43576 \rightarrow 12 \underline{6} 43578 \rightarrow 21 \underline{5} 43678 \rightarrow e
\end{aligned}
$$

(2) For each $r_{i, j} \neq r_{i, 1}\left(2 \leq i \leq k, 2 \leq j \leq k_{1}\right)$ in the cycle representation of $p$, a path $\pi\left(r_{i, j}\right)$ is constructed from $p$ to $e$ along which the $m$ misplaced symbols are 
corrected in order of the sequence $\left(r_{i, j}, r_{i, j+1}, \ldots, r_{i, k i}, C_{i+1}, \ldots, C_{k}, r_{1, k 1}, r_{1,1}\right.$, $\left.r_{1,2}, \ldots, r_{1, k 1-1}, C_{2}, \ldots, C_{i-1}, r_{i, 1}, r_{i, 2}, \ldots, r_{i, j-1}\right)$. The process results in $m-$ $(k-1)-\left|C_{1}\right|$ paths, each with length $m+k-3$.

$$
\begin{aligned}
\pi_{3}= & 21583476 \rightarrow 12 \underline{8} 53476 \rightarrow 21 \underline{6} 53478 \rightarrow 12453678 \rightarrow 21354678 \\
& \rightarrow 12 \underline{5} 34678 \rightarrow 21 \underline{4} 35678 \rightarrow e \\
\pi_{4}= & 21583476 \rightarrow 12 \underline{6} 83475 \rightarrow 21386475 \rightarrow 12 \underline{5} 86473 \rightarrow 21685473 \\
& \rightarrow 12 \underline{4} 85673 \rightarrow 21 \underline{8} 45673 \rightarrow e
\end{aligned}
$$

(3) For each $r_{i, j} \neq r_{i, 1}\left(i=1,3 \leq j \leq k_{1}, k_{1} \geq 3\right)$ in the cycle representation of $p$, a path $\pi\left(r_{i, j}\right)$ is constructed as follows:

Each path $\pi\left(r_{1, j}\right)$, with $3 \leq j \leq k_{1}-1$, is constructed along which $m$ misplaced symbols are corrected in order of the sequence $\left(r_{1, j}, r_{1, j+1}, \ldots, r_{1, k 1}, C_{2}, \ldots, C_{k}\right.$, $\left.r_{1,1}, r_{1,2}, \ldots, r_{1, j-1}\right)$.

Each path $\pi\left(r_{1, k 1}\right)$, with $k_{1} \geq 3$, is constructed along which $m$ misplaced symbols are corrected in order of the sequence $\left(r_{1, k 1}, C_{2}, \ldots, C_{k}, r_{1,2}, \ldots\right.$, $\left.r_{1, k 1-1}, r_{1,1}\right)$.

The processes outlined above yield $\left|C_{1}\right|-2$ paths, each of length $m+k-3$. Continuing with our example, since we have $k_{1}=2$, no path belonging this class exists.

(4) An $e_{j}$-path is generated by first moving a fixed element $e_{j}$ to position 3 , and then keeping $e_{j}$ away from its desired position until the other symbols have been corrected. Each such $e_{j}$-path, $\pi\left(e_{j}\right)$, is built in order of the correction sequence of $\left(e_{j}, C_{2}, \ldots, C_{k}, r_{1, k 1}, r_{1,1}, r_{1,2}, \ldots, r_{1, k 1-1}, e_{j}\right)$. The process outlined yields $n-$ $(m-2)-1=n-m+1$ paths, each of length $m+k-1$.

$$
\begin{aligned}
\pi_{5}= & 21583476 \rightarrow 52 \underline{1} 83476 \rightarrow 25 \underline{4} 83176 \rightarrow 52 \underline{8} 43176 \rightarrow 25 \underline{6} 43178 \\
& \rightarrow 52143678 \rightarrow 25341678 \rightarrow 32 \underline{5} 41678 \rightarrow 23 \underline{1} 45678 \rightarrow e \\
\pi_{6}= & 21583476 \rightarrow 15 \underline{2} 83476 \rightarrow 51 \underline{4} 83276 \rightarrow 15 \underline{8} 43276 \rightarrow 51 \underline{6} 43278 \\
& \rightarrow 15243678 \rightarrow 51342678 \rightarrow 13 \underline{5} 42678 \rightarrow 31 \underline{2} 45678 \rightarrow e \\
\pi_{7}= & 21583476 \rightarrow 12 \underline{7} 83456 \rightarrow 21 \underline{4} 83756 \rightarrow 12 \underline{8} 43756 \rightarrow 21 \underline{6} 43758 \\
\rightarrow & 12743658 \rightarrow 21347658 \rightarrow 12 \underline{5} 47638 \rightarrow 21 \underline{7} 45638 \rightarrow e
\end{aligned}
$$

B.3 The case of $\left\{p_{1}, p_{2}\right\} \cap\{1,2\}=\{r\}$, with $p_{1}=1$ or $p_{2}=2$

Each of the two subcases B3a and B3b of this case, corresponding to 3 and $r$ being in the same cycle or in different cycles, can be handled in a manner similar to that of Sect. B.1.

B.4 The case of $\left\{p_{1}, p_{2}\right\} \cap\{1,2\}=\{r\}$, with 1,2 in the same cycle $C_{i}$

Since 1 and 2 have the same function, we discuss the construction in terms of $r \in$ $\{1,2\}$. 
B4a. The subcase of 1, 2, 3 in the same cycle $C_{i}$

(1) For each head in the cycle representation of $p$, that is, $r_{i, 1}(1 \leq j \leq k)$, a path $\pi\left(r_{i, 1}\right)$ is constructed from $p$ to $e$ along which $m$ misplaced symbols are corrected in order of the sequence $\left(C_{i}, C_{i+1}, \ldots, C_{k}, C_{1}, C_{2}, \ldots, C_{i-1}\right)$, where the ordering is obtained by performing on $\left(C_{1}, C_{2}, \ldots, C_{k}\right)$ a number of left circular shifts which make the head of the cycle $C_{i}$ become the first element of the correction sequence. This process yields $k$ optimal paths of length $m+k-3$. For example, given $p=31247856=(213)(57)(68)(4)$ in $A N_{8}$, we construct these parallel paths:

$$
\begin{aligned}
\pi_{1}= & 31247856 \rightarrow 12347856 \rightarrow 21 \underline{5} 47836 \rightarrow 12 \underline{7} 45836 \rightarrow 21345876 \\
& \rightarrow 12 \underline{6} 45873 \rightarrow 21 \underline{8} 45673 \rightarrow e \\
\pi_{2}= & 31247856 \rightarrow 13 \underline{5} 47826 \rightarrow 31 \underline{7} 45826 \rightarrow 13245876 \rightarrow 31 \underline{6} 45872 \\
& \rightarrow 13 \underline{8} 45672 \rightarrow 31245678 \rightarrow e \\
\pi_{3}= & 31247856 \rightarrow 13 \underline{6} 47852 \rightarrow 31 \underline{8} 47652 \rightarrow 13247658 \rightarrow 21347658 \\
& \rightarrow 12 \underline{5} 47638 \rightarrow 21 \underline{7} 45638 \rightarrow e
\end{aligned}
$$

(2) For each $r_{i, j} \neq r_{i, 1}\left(2 \leq i \leq k, 2 \leq j \leq k_{i}\right)$ in the cycle representation of $p$, a path $\pi\left(r_{i, j}\right)$ is constructed from $p$ to $e$ along which the $m$ misplaced symbols are corrected in order of the sequence $\left(r_{i, j}, r_{i, j+1}, \ldots, r_{i, k i}, C_{i+1}, \ldots, C_{k}, r_{1, k 1}, r_{1,1}\right.$, $\left.r_{1,2}, \ldots, r_{1, k 1-1}, C_{2}, \ldots, C_{i-1}, r_{i, 1}, r_{i, 2}, \ldots, r_{i, j-1}\right)$. From this process, we have $m-(k-1)-\left|C_{1}\right|$ paths, each of length $m+k$. For our example:

$$
\begin{aligned}
\pi_{4}= & 31247856 \rightarrow 13 \underline{7} 42856 \rightarrow 31542876 \rightarrow 13 \underline{6} 42875 \rightarrow 31 \underline{8} 42675 \\
& \rightarrow 13542678 \rightarrow 51 \underline{3} 42678 \rightarrow 15 \underline{2} 43678 \rightarrow 21 \underline{5} 43678 \rightarrow e \\
\pi_{5}= & 31247856 \rightarrow 13 \underline{8} 47256 \rightarrow 31647258 \rightarrow 16 \underline{3} 47258 \rightarrow 61 \underline{2} 47358 \\
& \rightarrow 12647358 \rightarrow 21 \underline{5} 47368 \rightarrow 12 \underline{7} 45368 \rightarrow 21 \underline{6} 45378 \rightarrow e
\end{aligned}
$$

(3) For each $r_{i, j} \neq r_{i, 1}\left(i=1,3 \leq j \leq k_{1}, k_{1} \geq 3\right)$ in the cycle representation of $p$, a path $\pi\left(r_{i, j}\right)$ is constructed as follows; note that the path $\pi\left(r_{1,1}\right)$ is the same as the path $\pi\left(r_{1,2}\right)$ :

Each path $\pi\left(r_{1, j}\right)$, with $3 \leq j \leq k_{1}-1$, is constructed along which $m$ misplaced symbols are corrected in order of the sequence $\left(r_{1, j}, r_{1, j+1}, \ldots, r_{1, k 1}\right.$, $\left.C_{2}, \ldots, C_{k}, r_{1,1}, r_{1,2}, \ldots, r_{i, j-1}\right)$.

Each path $\pi\left(r_{1, k 1}\right)$, with $k_{1} \geq 3$, is constructed along which $m$ misplaced symbols are corrected in order of the sequence $\left(r_{1, k 1}, C_{2}, \ldots, C_{k}, r_{1,2}, \ldots, r_{1, k 1-1}\right.$, $\left.r_{1,1}\right)$.

The process above yields $\left|C_{1}\right|-2$ paths, each of length $m+k-1$. For our example, we have:

$$
\begin{aligned}
\pi_{6}= & 31247856 \rightarrow 23 \underline{1} 47856 \rightarrow 32 \underline{5} 47816 \rightarrow 23 \underline{7} 45816 \\
& \rightarrow 32145876 \rightarrow 23 \underline{6} 45871 \rightarrow 32 \underline{8} 45671 \rightarrow 23 \underline{1} 45678 \rightarrow e
\end{aligned}
$$


(4) An $e_{j}$-path is generated by first moving a fixed element $e_{j}$ to position 3 , and then keeping $e_{j}$ away from its desired position until the other symbols have been corrected. Such an $e_{j}$-path, $\pi\left(e_{j}\right)$, is constructed in order of the correction sequence $\left(e_{j}, C_{2}, \ldots, C_{k}, r_{1, k 1}, r_{1,1}, r_{1,2}, \ldots, r_{1, k 1-1}, e_{j}\right)$. From this process, we obtain $n-m$ paths, each of length $m+k+1$. For our example, we get:

$$
\begin{aligned}
\pi_{7}= & 31247856 \rightarrow 13 \underline{4} 27856 \rightarrow 31 \underline{5} 27846 \rightarrow 13 \underline{72} 25846 \rightarrow 31425876 \\
& \rightarrow 13 \underline{6} 25874 \rightarrow 31 \underline{8} 25674 \rightarrow 13425678 \rightarrow 41325678 \rightarrow 14235678 \\
& \rightarrow 21 \underline{4} 35678 \rightarrow e
\end{aligned}
$$

B4b. The subcase of 3 not in the cycle $C_{i}$ that contains 1,2

(1) For each head in the cycle representation of $p$, that is, $r_{i, 1}(1 \leq i \leq k)$, a path $\pi\left(r_{i, 1}\right)$ is constructed from $p$ to $e$ along which $m$ misplaced symbols are corrected in order of the sequence $\left(C_{i}, C_{i+1}, \ldots, C_{k}, C_{1}, C_{2}, \ldots, C_{i-1}\right)$, where the ordering is obtained by performing on $\left(C_{1}, C_{2}, \ldots, C_{k}\right)$ a number of left circular shifts which make the head of the cycle $C_{i}$ become the first element of the correction sequence. From this process, we obtain $k$ optimal paths of length $m+k-3$. For example, given $p=25841763=(83)(512)(67)(4)$ in $A N_{8}$, we construct these parallel paths:

$$
\begin{aligned}
\pi_{1}= & 25 \underline{8} 41763 \rightarrow 52341768 \rightarrow 23 \underline{5} 41768 \rightarrow 32145768 \rightarrow 21345768 \\
& \rightarrow 12 \underline{6} 45738 \rightarrow 21 \underline{7} 45638 \rightarrow e \\
\pi_{2}= & 25841763 \rightarrow 82 \underline{5} 41763 \rightarrow 28145763 \rightarrow 8 \underline{6} \underline{6} 45713 \rightarrow 28 \underline{7} 45613 \\
& \rightarrow 82145673 \rightarrow 21 \underline{8} 45673 \rightarrow e \\
\pi_{3}= & 25841763 \rightarrow 52 \underline{6} 41783 \rightarrow 25 \underline{7} 41683 \rightarrow 52 \underline{8} 41673 \rightarrow 25341678 \\
& \rightarrow 32 \underline{5} 41678 \rightarrow 23145678 \rightarrow e
\end{aligned}
$$

(2) For each $r_{i, j} \neq r_{i, 1}\left(2 \leq i \leq k, 2 \leq j \leq k_{i}\right)$ in the cycle representation of $p$, a path $\pi\left(r_{i, j}\right)$ is constructed from $p$ to $e$ along which $m$ misplaced symbols are corrected in order of the sequence $\left(r_{i, j}, r_{i, j+1}, \ldots, r_{i, k i}, C_{i+1}, \ldots, C_{k}, r_{1, k 1}, r_{1,1}\right.$, $\left.r_{1,2}, \ldots, r_{1, k 1-1}, C_{2}, \ldots, C_{i-1}, r_{i, 1}, r_{i, 2}, \ldots, r_{i, j-1}\right)$. We thus obtain $m-(k-$ 1) $-\left|C_{1}\right|$ paths, each of length $m+k-1$. For our example, we have:

$$
\begin{aligned}
\pi_{4}= & 25841763 \rightarrow 52 \underline{7} 41863 \rightarrow 25641873 \rightarrow 52341876 \rightarrow 25 \underline{8} 41376 \\
& \rightarrow 52641378 \rightarrow 26 \underline{5} 41378 \rightarrow 62145378 \rightarrow 21 \underline{6} 45378 \rightarrow e \\
\pi_{5}= & 25841763 \rightarrow 52148763 \rightarrow 25 \underline{6} 48713 \rightarrow 52 \underline{7} 48613 \rightarrow 25148673 \\
& \rightarrow 52348671 \rightarrow 25 \underline{8} 43671 \rightarrow 52143678 \rightarrow 21 \underline{5} 43678 \rightarrow e
\end{aligned}
$$

(3) For each $r_{i, j} \neq r_{i, 1}\left(i=1,3 \leq j \leq k_{1}, k_{1} \geq 3\right)$ in the cycle representation of $p$, a path $\pi\left(r_{i, j}\right)$ is constructed as follows: 
Each path $\pi\left(r_{1, j}\right)$, with $3 \leq j \leq k_{1}-1$, is constructed along which $m$ misplaced symbols are corrected in order of the sequence $\left(r_{1, j}, r_{1, j+1}, \ldots, r_{1, k 1}\right.$, $\left.C_{2}, \ldots, C_{k}, r_{1,1}, r_{1,2}, \ldots, r_{1, j-1}\right)$.

Each path $\pi\left(r_{1, k 1}\right)$, with $k_{1} \geq 3$, is constructed along which $m$ misplaced symbols are corrected in order of the sequence $\left(r_{1, k 1}, C_{2}, \ldots, C_{k}, r_{1,2}, \ldots\right.$, $\left.r_{1, k 1-1}, r_{1,1}\right)$.

From the above, we obtain $\left|C_{1}\right|-2$ paths, each of length $m+k-1$. Since $k_{1}=2$ in our example, there exists no path in this class.

(4) An $e_{j}$-path is generated by first moving a fixed element $e_{j}$ to position 3 , and then keeping $e_{j}$ away from its desired position until the other symbols have been corrected. Such an $e_{j}$-path, $\pi\left(e_{j}\right)$, is constructed in the order of the correction sequence $\left(e_{j}, C_{2}, \ldots, C_{k}, r_{1, k 1}, r_{1,1}, r_{1,2}, \ldots, r_{1, k 1-1}, e_{j}\right)$. As a result, we have $n-m-1$ paths, each of length $m+k+2$. For our example, the paths are:

$$
\begin{aligned}
\pi_{6}= & 25841763 \rightarrow 58 \underline{2} 41763 \rightarrow 85 \underline{6} 41723 \rightarrow 58 \underline{7} 41623 \rightarrow 85241673 \\
& \rightarrow 58341672 \rightarrow 35 \underline{8} 41672 \rightarrow 53241678 \rightarrow 35142678 \rightarrow 13542678 \\
& \rightarrow 31 \underline{2} 45678 \rightarrow e \\
\pi_{7}= & 25841763 \rightarrow 52 \underline{4} 81763 \rightarrow 25 \underline{6} 81743 \rightarrow 52 \underline{78} 81643 \rightarrow 25481673 \\
& \rightarrow 52381674 \rightarrow 25 \underline{8} 31674 \rightarrow 52431678 \rightarrow 24 \underline{5} 31678 \rightarrow 42135678 \\
& \rightarrow 21 \underline{43} 35678 \rightarrow e
\end{aligned}
$$

B.5 The case of $\left\{p_{1}, p_{2}\right\} \cap\{1,2\}=\{r\}$, with 1 and 2 in different cycles

Each of the two subcases B5a and B5b of this case, corresponding to 3 and $r$ being in the same cycle or in different cycles, can be handled in a manner similar to that of Sect. B.4.

B.6 The case of $\left\{p_{1}, p_{2}\right\} \cap\{1,2\}=\Phi$, with 1 and 2 in the same cycle $C_{i}$

B6a. The subcase of 1, 2, 3 in the same cycle $C_{i}$

(1) For each head in the cycle representation of $p$, that is, $r_{i, 1}(1 \leq i \leq k)$, a path $\pi\left(r_{i, 1}\right)$ is constructed from $p$ to $e$ along which $m$ misplaced symbols are corrected in order of the sequence $\left(C_{i}, C_{i+1}, \ldots, C_{k}, C_{1}, C_{2}, \ldots, C_{i-1}\right)$, where the ordering sequence is obtained by performing on $\left(C_{1}, C_{2}, \ldots, C_{k}\right)$ a number of left circular shifts which make the head of the cycle $C_{i}$ become the first element of the correction sequence. This process yields $k$ optimal paths of length $m+k-2$. For example, given $p=3526147=(2513)(46)(7)$ in $A N_{7}$, we obtain:

$$
\begin{aligned}
\pi_{1}= & 3526147 \rightarrow 23 \underline{5} 6147 \rightarrow 3216547 \rightarrow 2136547 \rightarrow 12 \underline{46537} \\
& \rightarrow 21 \underline{6} 4537 \rightarrow e \\
\pi_{2}= & 3526147 \rightarrow 53 \underline{4} 6127 \rightarrow 35 \underline{6} 4127 \rightarrow 5324167 \rightarrow 32 \underline{5} 4167 \\
& \rightarrow 2314567 \rightarrow e
\end{aligned}
$$


(2) For each $r_{i, j} \neq r_{i, 1}\left(2 \leq i \leq k, 2 \leq j \leq k_{i}\right)$ in the cycle representation of $p$, a path $\pi\left(r_{i, j}\right)$ is constructed from $p$ to $e$ along which the $m$ misplaced symbols are corrected in order of the sequence $\left(r_{i, j}, r_{i, j+1}, \ldots, r_{i, k i}, C_{i+1}, \ldots, C_{k}, r_{1, k 1}, r_{1,1}\right.$, $\left.r_{1,2}, \ldots, r_{1, k 1-1}, C_{2}, \ldots, C_{i-1}, r_{i, 1}, r_{i, 2}, \ldots, r_{i, j-1}\right)$. The results of this process are $m-(k-1)-\left|C_{1}\right|$ paths, each of length $m+k$. For our example:

$$
\begin{aligned}
\pi_{3}= & 3526147 \rightarrow 53 \underline{6} 2147 \rightarrow 6532147 \rightarrow 5623147 \rightarrow 62 \underline{5} 3147 \rightarrow 26 \underline{13547} \\
& \rightarrow 1263547 \rightarrow 21 \underline{4} 3567 \rightarrow e
\end{aligned}
$$

(3) For each $r_{i, j} \neq r_{i, 1}\left(i=1,3 \leq j \leq k_{1}, k_{1} \geq 3\right)$ in the cycle representation of $p$, a path $\pi\left(r_{i, j}\right)$ is constructed as follows:

Each path $\pi\left(r_{1, j}\right)$, with $3 \leq j \leq k_{1}-1$, is constructed along which $m$ misplaced symbols are corrected in order of the sequence $\left(r_{1, j}, r_{1, j+1}, \ldots, r_{1, k 1}\right.$, $\left.C_{2}, \ldots, C_{k}, r_{1,1}, r_{1,2}, \ldots, r_{1, j-1}\right)$.

Each path $\pi\left(r_{1, k 1}\right)$, with $k_{1} \geq 3$, is constructed along which $m$ misplaced symbols are corrected in order of the sequence $\left(r_{1, k 1}, C_{2}, \ldots, C_{k}, r_{1,2}, \ldots, r_{1, k 1-1}\right.$, $\left.r_{1,1}\right)$.

From this process, we have $\left|C_{1}\right|-2$ paths, each of length $m+k$. For our example:

$$
\begin{aligned}
\pi_{4}= & 3526147 \rightarrow 5316247 \rightarrow 1536247 \rightarrow 5126347 \rightarrow 15 \underline{4} 6327 \rightarrow 51 \underline{6} 4327 \\
& \rightarrow 1524367 \rightarrow 21 \underline{5} 4367 \rightarrow e \\
\pi_{5}= & 3526147 \rightarrow 5236147 \rightarrow 25 \underline{4} 6137 \rightarrow 52 \underline{6} 4137 \rightarrow 26 \underline{5} 4137 \rightarrow 6214537 \\
& \rightarrow 1624537 \rightarrow 2164537 \rightarrow e
\end{aligned}
$$

(4) An $e_{j}$-path is generated by first moving a fixed element $e_{j}$ to position 3 , and then keeping $e_{j}$ away from its desired position until the other symbols have been corrected. Such an $e_{j}$-path, $\pi\left(e_{j}\right)$, is constructed in order of the correction sequence $\left(e_{j}, C_{2}, \ldots, C_{k}, r_{1, k 1}, r_{1,1}, r_{1,2}, \ldots, r_{1, k 1-1}, e_{j}\right)$. The results are $n-m$ paths, each of length $m+k+2$. For our example, we obtain:

$$
\begin{aligned}
\pi_{6}= & 3526147 \rightarrow 53 \underline{7} 6142 \rightarrow 35 \underline{4} 6172 \rightarrow 53 \underline{6} 4172 \rightarrow 3574172 \rightarrow 5734162 \\
& \rightarrow 7524162 \rightarrow 27 \underline{5} 4163 \rightarrow 7214563 \rightarrow 21 \underline{7} 4563 \rightarrow e
\end{aligned}
$$

B6b. The subcase of 3 not in the cycle $C_{i}$ that contains 1,2

(1) For each head in the cycle representation of $p$, that is $r_{i, 1}(1 \leq i \leq k)$, a path $\pi\left(r_{i, 1}\right)$ is constructed from $p$ to $e$ along which $m$ misplaced symbols are corrected in order of the sequence $\left(C_{i}, C_{i+1}, \ldots, C_{k}, C_{1}, C_{2}, \ldots, C_{i-1}\right)$, where the ordering sequence is obtained by performing on $\left(C_{1}, C_{2}, \ldots, C_{k}\right)$ a number of left circular shifts which make the head of the cycle $C_{i}$ become the first element of the correction sequence. As a result of this process, we obtain $k$ optimal paths of length $m+k-2$. For example, given $p=4572163=(73)(4251)(6)$ in $A N_{7}$, 
we construct these parallel paths:

$$
\begin{aligned}
\pi_{1}= & 45 \underline{7} 2163 \rightarrow 5432167 \rightarrow 35 \underline{4} 2167 \rightarrow 5324167 \rightarrow 32 \underline{5} 4167 \\
& \rightarrow 2314567 \rightarrow e \\
\pi_{2}= & 4572163 \rightarrow 57 \underline{4} 2166 \rightarrow 7524163 \rightarrow 27 \underline{5} 4163 \rightarrow 7214563 \\
& \rightarrow 21 \underline{7} 4563 \rightarrow e
\end{aligned}
$$

(2) For each $r_{i, j} \neq r_{i, 1}\left(2 \leq i \leq k, 2 \leq j \leq k_{i}\right)$ in the cycle representation of $p$, a path $\pi\left(r_{i, j}\right)$ is constructed from $p$ to $e$ along which the $m$ misplaced symbols are corrected in order of the sequence $\left(r_{i, j}, r_{i, j+1}, \ldots, r_{i, k i}, C_{i+1}, \ldots, C_{k}, r_{1, k 1}, r_{1,1}\right.$, $\left.r_{1,2}, \ldots, r_{1, k 1-1}, C_{2}, \ldots, C_{i-1}, r_{i, 1}, r_{i, 2}, \ldots, r_{i, j-1}\right)$. This process yields $m-$ $(k-1)-\left|C_{1}\right|$ paths, each of length $m+k$. For our example, we have:

$$
\begin{aligned}
\pi_{3}= & 4572163 \rightarrow 5427163 \rightarrow 42 \underline{5} 7163 \rightarrow 2417563 \rightarrow 1247563 \rightarrow 2137564 \\
& \rightarrow 12 \underline{7} 3564 \rightarrow 21 \underline{4} 3567 \rightarrow e \\
\pi_{4}= & 4572163 \rightarrow 74 \underline{5} 2163 \rightarrow 4712563 \rightarrow 7142563 \rightarrow 1732564 \rightarrow 31 \underline{72} 564 \\
& \rightarrow 13 \underline{4} 2567 \rightarrow 3124567 \rightarrow e \\
\pi_{5}= & 4572163 \rightarrow 5412763 \rightarrow 1542763 \rightarrow 5132764 \rightarrow 15 \underline{72} 2364 \rightarrow 51 \underline{4} 2367 \\
& \rightarrow 1524367 \rightarrow 21 \underline{5} 4367 \rightarrow e
\end{aligned}
$$

(3) For each $r_{i, j} \neq r_{i, 1}\left(i=1,3 \leq j \leq k_{1}, k_{1} \geq 3\right)$ in the cycle representation of $p$, a path $\pi\left(r_{i, j}\right)$ is constructed as follows; note that the path $\pi\left(r_{1,1}\right)$ is the same as the path $\pi\left(r_{1,2}\right)$ :

Each path $\pi\left(r_{1, j}\right)$, with $3 \leq j \leq k_{1}-1$, is constructed along which $m$ misplaced symbols are corrected in order of the sequence $\left(r_{1, j}, r_{1, j+1}, \ldots, r_{1, k 1}\right.$, $\left.C_{2}, \ldots, C_{k}, r_{1,1}, r_{1,2}, \ldots, r_{1, j-1}\right)$.

Each path $\pi\left(r_{1, k 1}\right)$, with $k_{1} \geq 3$, is constructed along which $m$ misplaced symbols are corrected in order of the sequence $\left(r_{1, k 1}, C_{2}, \ldots, C_{k}, r_{1,2}, \ldots\right.$, $\left.r_{1, k 1-1}, r_{1,1}\right)$.

From the above, we have $\left|C_{1}\right|-2$ paths, each of length $m+k-1$. Since $k_{1}=2$ in our example, there exists no path in this class.

(4) An $e_{j}$-path is generated by first moving a fixed element $e_{j}$ to position 3 , and then keeping $e_{j}$ away from its desired position until the other symbols have been corrected. Such an $e_{j}$-path, $\pi\left(e_{j}\right)$, is constructed in order of the correction sequence $\left(e_{j}, C_{2}, \ldots, C_{k}, r_{1, k 1}, r_{1,1}, r_{1,2}, \ldots, r_{1, k 1-1}, e_{j}\right)$. From this process, we obtain $n-m$ paths, each of length $m+k+2$. For our example:

$$
\begin{aligned}
\pi_{6}= & 4572163 \rightarrow 54 \underline{6} 2173 \rightarrow 65 \underline{4} 2173 \rightarrow 5624173 \rightarrow 62 \underline{5} 4173 \rightarrow 2614573 \\
& \rightarrow 1264573 \rightarrow 2134576 \rightarrow 12 \underline{7} 4536 \rightarrow 21 \underline{6} 4537 \rightarrow e
\end{aligned}
$$

This concludes the final part of the proof of Theorem 1, corresponding to the condition $p_{3} \neq 3$; the complementary part, associated with $p_{3}=3$ was covered in Appendix A. 


\section{References}

1. Akers SB, Krishnamurthy B (1989) A group-theoretic model for symmetric interconnection networks. IEEE Trans Comput 38(4):555-566

2. Akers SB, Harel D, Krishnamurthy B (1987) The star graph, an attractive alternative to the $n$-cube. In: Proc. int'l conf parallel processing, 1987, pp 393-400

3. Bhuyan LN, Agrawal DP (1984) Generalized hypercube and hyperbus structures for a computer network. IEEE Trans Comput 33:323-333

4. Chen B, Xiao WJ, Parhami B (2006) Internode distance and optimal routing in a class of alternating group networks. IEEE Trans Comput 12(12):1645-1648

5. Day K, Al-Ayyoub AE (1997) Fault diameter of $k$-ary $n$-cube networks. IEEE Trans Parallel Distrib Syst 8(9):903-907

6. Day K, Tripathi A (1994) A comparative study of topological properties of hypercubes and star graphs. IEEE Trans Parallel Distrib Syst 5(1):31-38

7. Du DZ, Hsu DF (1996) Combinatorial network theory. Kluwer Academic, Dordrecht

8. Duh DR, Chen GH, Hsu DF (1996) Combinatorial properties of generalized hypercube graphs. Inf Process Lett 57:141-145

9. Flandrin E, Li H (1994) Mengerian properties, Hamiltonicity, and claw-free graphs. Networks 4:177183

10. Fu JS, Chen GH, Duh DR (2002) Vertex-disjoint paths and related problems on hierarchical networks. Networks 40(10):142-154

11. Gu QP, Peng S (1997) Vertex to set disjoint paths problem in star graph. Inf Process Lett 62(4):201207

12. Gupta AK, Dally WJ (2006) Topology optimization of interconnection networks. IEEE Comput Archit Lett 5(1):10-13

13. Haddadi H, Iannaccone G, Moore A, Mortier R, Rio M (2008) A survey on network topology: inference, modelling and generation. IEEE Commun Surv 10(2):48-69

14. Hsu DF (1994) On container width and length in graphs, groups, and networks. IEICE Trans Fundam E77-A:668-680

15. Ishiyami Y (1996) The wide-diameter of the $n$-dimensional toroidal mesh networks. Networks 27:257-266

16. Jwo JS, Lakshmivarahan S, Dhall SK (1993) A new class of interconnection networks based on the alternating groups. Networks 23:315-326

17. Lakshmivarahan S, Jwo JS, Dhall SK (1993) Symmetry in interconnection networks based on Cayley graphs of permutations-a survey. Parallel Comput 19:361-407

18. Liaw SC, Chang GJ (1998) Generalized diameters and Rabin numbers of networks. J Comb Optim 2(4):371-384

19. Lin T-C, Duh D-R (2008) Constructing vertex-disjoint paths in $(n, k)$-star graphs. Inf Sci 178:788801

20. Liszka KJ, Antonio JK, Siegel HJ (1997) Problems with comparing interconnection networks: is an alligator better than an armadillo? IEEE Concurr 5(4):18-28

21. Lua EK, Crowcroft J, Pias M, Sharma R, Lim S (2005) A survey and comparison of peer-to-peer overlay network schemes. IEEE Commun Surv 7(2):72-93

22. Owens JD, Dally WJ, Ho R, Jayasimha DN, Keckler SW, Peh L-S (2007) Research challenges for on-chip interconnection networks. IEEE Micro 27(5):96-108

23. Parhami B (1999) Introduction to parallel processing: algorithms and architectures. Plenum Press, New York

24. Suzuki Y, Kaneko K (2006) An algorithm for disjoint paths in bubble-sort graphs. Syst Comput Jpn 37(12):27-32

25. Xu J (2001) Topological structure and analysis of interconnection networks. Kluwer Academic, Dordrecht

26. Xu J (2004) Wide Diameters of Cartesian product graphs and digraphs. J Comb Optim 8:171-181

27. Youhu J (1998) A class of Cayley networks based on the alternating groups. Adv Math (Chin) 4:361362 

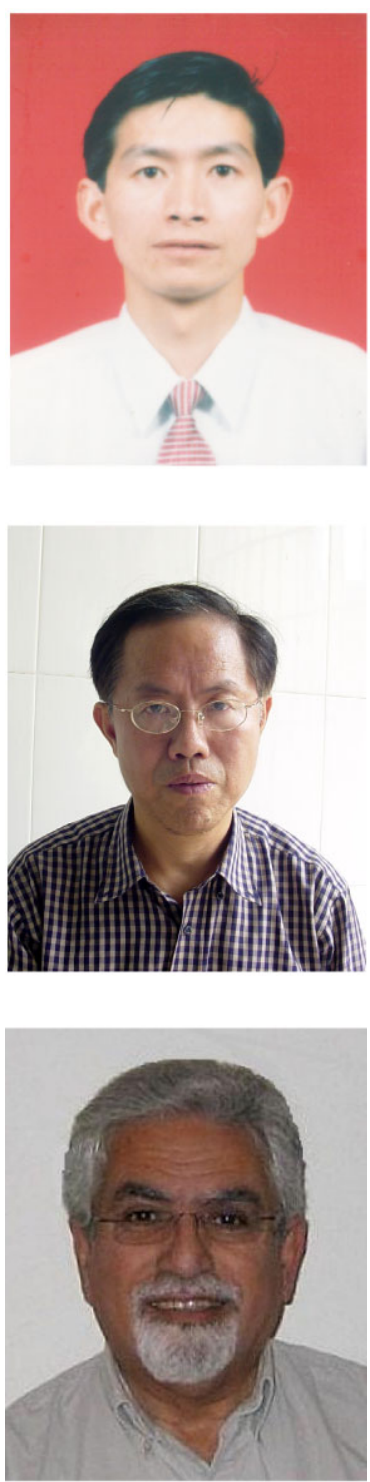

Wenjun Xiao received the Ph.D. degree in Mathematics from Sichuan University, People's Republic of China, in 1989. Currently, he is Professor in the School of Computer Science and Engineering, South China University of Technology, Guangzhou, People's Republic of China. His research interests include discrete mathematics, parallel and distributed computing, complex networks, and software architecture. He has published more than 60 papers in international conferences and journals, including IEEE Trans. Computers and IEEE Trans. Parallel and Distributed Systems on these topics since 1985.

Behrooz Parhami received the Ph.D. degree in Computer Science from University of California, Los Angeles, in 1973. Currently, he is Professor in the Department of Electrical and Computer Engineering, University of California, Santa Barbara, USA. His research deals with parallel architectures and algorithms, computer arithmetic, and reliable computing. In his previous position with Sharif University of Technology in Tehran, Iran (1974-1988), he was also involved in the areas of educational planning, curriculum development, standardization efforts, technology transfer, and various editorial responsibilities, including a fiveyear term as Editor of Computer Report, a Persian-language computing periodical. Doctor Parhami's technical publications include more than 250 papers in journals and international conferences, a Persianlanguage textbook, and an English/Persian glossary of computing terms. Among his latest publications are two graduate-level textbooks on parallel processing (Plenum, 1999) and computer arithmetic (Oxford, 2000), and an introductory textbook on computer architecture (Oxford, 2005). Doctor Parhami serves as Fellow of the IEEE and the IEEE Computer Society, Chartered Fellow of the British Computer Society, Member of the ACM, and Distinguished Member of the Informatics Society of Iran, for which he served as Founding Member and President during 1979-1984. He also served as Chairman of the IEEE Iran Section (1977-1986) and received the IEEE Centennial Medal in 1984. He currently serves as Associate Editor of both IEEE Trans. Computers and IEEE Trans. Parallel and Distributed Systems. 